\title{
REVISIÓN SISTEMÁTICA DE LA LITERATURA DE LA AUTOMATIZACIÓN DE PROCESOS DE NEGOCIO
}

\author{
Abilio Tinoco León \\ tinoco@bpi-center.com \\ BPI Center. Lima, Perú
}

Las empresas enfrentan el desafío de colocar un producto o servicio antes que su competencia, y necesitan responder a los requerimientos de sus clientes; por estos motivos, su estructura organizacional está orientándose a una optimización de los procesos de negocio. Se requiere que estos sean ágiles para responder al entorno empresarial, es decir, a los requerimientos del cliente, así como a las nuevas disposiciones legales y a las estrategias del negocio. La automatización de procesos de negocio contribuye significativamente con la agilidad de estos procesos. El presente artículo tiene el propósito de clasificar los métodos utilizados para la automatización de procesos de negocio. La revisión literaria utilizó como referencia artículos relacionados con este tema. Por tanto, se buscaron artículos en Scopus, Google Scholar y Springer. El proceso de revisión se realizó en dos etapas: en la primera los artículos encontrados se filtraron sobre la base de la lectura de los títulos, resúmenes y palabras claves; y en la segunda se seleccionaron los artículos en base a la lectura completa de los artículos filtrados.

Palabras clave: gestión de procesos de negocio (BPM) / sistema de gestión de reglas de negocio (BRMS) / arquitectura dirigida por modelos (MDA)

\section{Abstract}

\section{Systematic review of the automation of business processes' literature}

Companies face the challenge of placing a product or service before their competition, and need to respond to their customers' requirements. For these reasons, its organizational structure is being oriented to an optimization of the business processes. They are required to be agile to respond to the business environment, i.e., customer requirements, as well as new legal provisions and business strategies. The automation of business processes contributes significantly with the agility of these processes. The purpose of this article is to classify the methods used for the automation of business processes. The literary review used articles related to this topic as reference. Therefore, articles were searched in Scopus, Google Scholar and Springer. The review process was carried out in two stages: first, the articles found were filtered based on the reading of the titles, abstracts and keywords; second, articles were selected based on the complete reading of the filtered articles.

Keywords: business process management (BPM) / business rule management system (BRMS) / model driven architecture (MDA) 


\section{Introducción}

Las empresas enfrentan el desafío de la competitividad, donde los recursos claves son los procesos de negocio (Lederer, Schott, Huber y Kurz, 2013), los cuales son un conjunto estructurado y medible de actividades diseñadas para conseguir un resultado concreto para algún cliente o mercado específico (Davenport, 1993); así como la agilidad de dichos procesos (Heininger, 2012), entendiéndose por agilidad a la posibilidad de añadir o reconfigurar capacidades de procesos nuevos o mejorados; haciendo hincapié en el tiempo y la habilidad de las personas para recuperarse del cambio, y de adaptarse a dichas situaciones; es la comprensión del proceso de principio a fin (Heininger, 2012).

La automatización de procesos de negocio se refiere a los procesos o funciones de negocio complejos, más allá de las actividades de manipulación de datos y mantenimiento de registros, mediante el uso de tecnologías avanzadas. Se centra en la operación del negocio en vez de la demanda, y en los procesos principales de misión crítica. El fracaso de los proyectos de automatización se debe a que se inician desde una perspectiva tecnológica y no de negocio (Meertens, lacob, Nieuwenhuis, Van Sinderen, Jonkers, y Quartel, 2012). Para enfrentar el desafío de la competitividad, el estilo de gestión empresarial es crítico: enfocada al cliente, alineada estratégicamente y centrada en procesos (Burlton, 2010), y la automatización de los procesos negocio orientado a servicios (Gulledge, 2015). Se han realizado esfuerzos para que los procesos apoyen al logro de objetivos; por ejemplo, Markus (2010) propone mecanismos de gobierno de procesos de negocio; Gulledge (2015) la implementación orientada a servicios alineado con la tecnología; Scheer y Brabänder (2010) una infraestructura organizacional, roles y responsabilidades para el proceso de BPM; y Burlton (2001) un método para articular medio ambiente, intención, stakeholders, estrategias, procesos de negocio y otras capacidades, y sus relaciones entre ellos con integridad.

El presente artículo recopila y organiza información sobre la automatización de procesos de negocio, con la finalidad de analizar las tendencias y variabilidad de los elementos del proceso de automatización. En la sección 2 se describe la metodología de investigación; en la sección 3 se presenta la taxonomía de procesos de automatización; en la sección 4 se hace el análisis de revisión literaria, y se finaliza con las conclusiones.

\section{Metodología de investigación}

El proceso de revisión literaria se esquematiza en la figura 1: 
Figura 1. Proceso de investigación

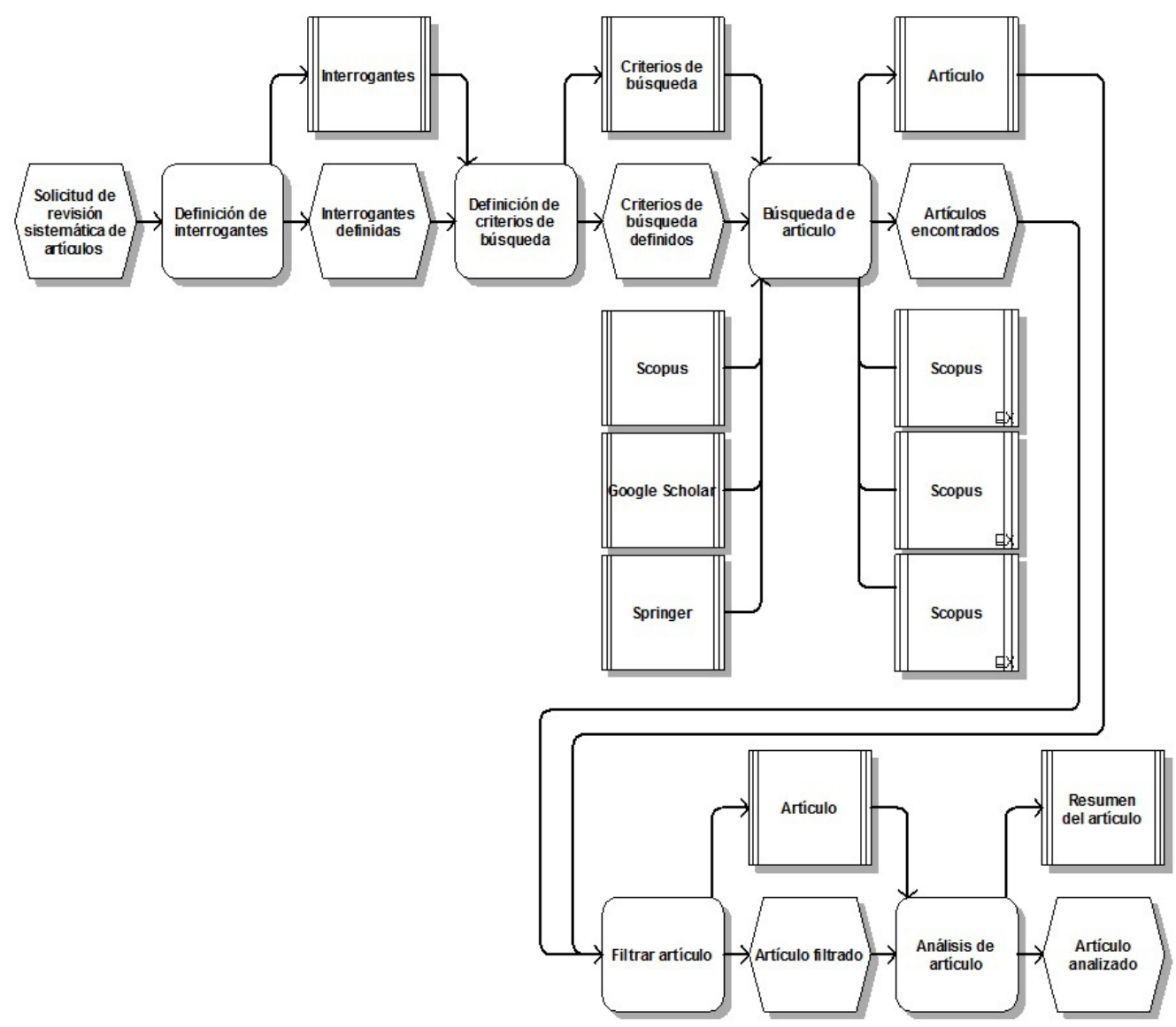

Elaboración propia

Un aspecto importante del proceso señalado se refiere a las interrogantes planteadas, las cuales se detallan a continuación:

i. Q1 ¿Cuáles son las actividades del ciclo de vida de la automatización de procesos de negocio?

ii. Q2 ¿Cuál es el nivel de integración entre las actividades del ciclo de vida de la automatización de procesos de negocio?

iii. Q3 ¿Qué métodos de automatización de procesos de negocio se usan en cada una de las actividades del proceso de automatización de procesos de negocio? 
iv. Q4 ¿Qué modelos de procesos de negocio se usan en cada una de las actividades del proceso de automatización de procesos de negocio?

v. Q5 ¿Qué objetivos e indicadores se usan en el proceso de automatización de procesos de negocio y en cada una de las actividades del proceso de automatización de los procesos de negocio?

Las respuestas a estas interrogantes ayudarán a identificar los elementos y relaciones de un proceso de automatización de procesos de negocio.

\section{Estadísticas}

La figura 2 muestra la distribución por año de los 3966 documentos relacionados con el Business Process Automation encontrados en Scopus, entre el año 1969 y el 2015.

Figura 2. Documentos relacionados con Business Process Automation

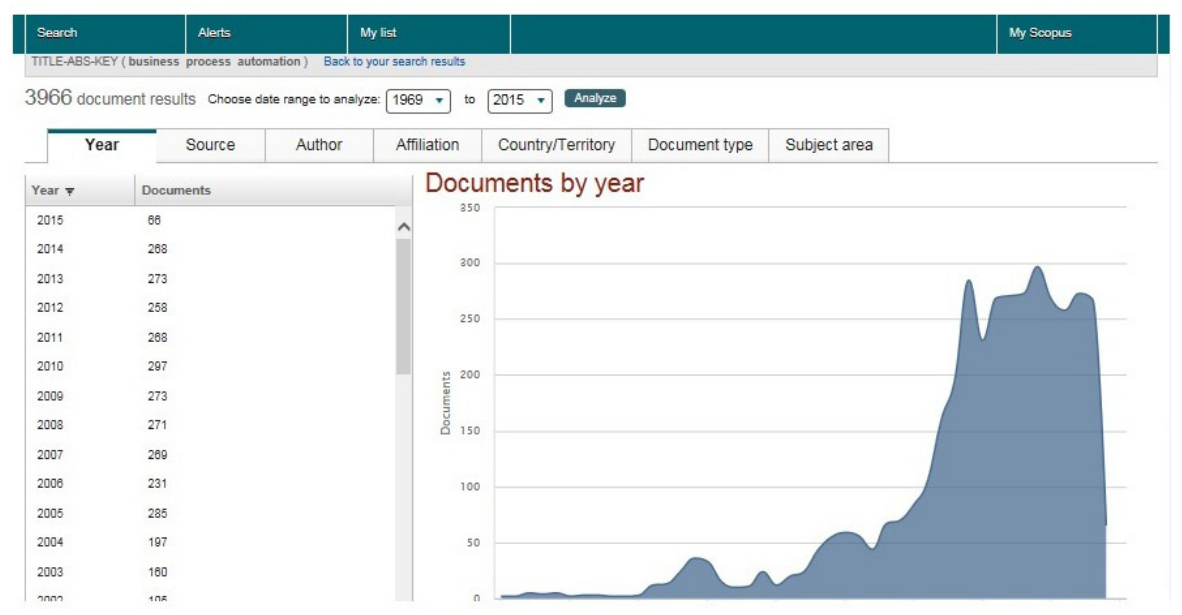

Fuente: captura de pantallas Scopus

\section{Análisis}

El análisis de la revisión literaria está enfocado a responder a las interrogantes:

\subsection{Q1 ¿Cuáles son las actividades del proceso de automatización de procesos de negocio?}

El ciclo de vida de la Gestión de Procesos de Negocio es el conjunto de pasos o actividades que se deben realizar para llevar a cabo un proyecto de Gestión de Procesos de Negocio, donde algunas de las actividades son ejecutadas como parte del proceso de automatización de procesos de negocio. La tipología de ciclo de vida de Gestión 
de Procesos de Negocio propuesto por Macedo, Kazan, Dallavalle y Lucirton (2014) fue actualizado considerando a El Kharbili y Keil (2010), Šaša (2010), Gong y Janssen (2012), Tran, Zdun, Holmes Oberortner, Mulo y Dustdar (2012), Isaca (2012), Ndie, Tangha y Ekwoge (2010), Scheer y Brabänder (2010), Duarte, Machado y Fernandes (2012), Fabra, De Castro, Álvarez y Marcos (2012), Lederer et al. (2013), Santos, Duarte, Machado y Fernandes (2013), y Gulledge (2015). Los resultados se pueden ver en la tabla 1.

\section{Tabla 1. Tipología de ciclo de vida de gestión de procesos de negocio}

\begin{tabular}{|c|c|c|c|c|c|c|c|}
\hline Referencia & & & & Actividades & & & \\
\hline $\begin{array}{l}\text { ABPMP } \\
(2009)\end{array}$ & $\begin{array}{c}\text { No es actividad } \\
\text { de ABPMP } \\
(2009)\end{array}$ & $\begin{array}{l}\text { Planeamiento } \\
\text { y estrategia }\end{array}$ & Análisis & $\begin{array}{c}\text { Diseño y } \\
\text { modelamiento }\end{array}$ & Implementación & $\begin{array}{l}\text { Monitoreo } \\
\text { y control }\end{array}$ & Refinamiento \\
\hline $\begin{array}{l}\text { Hallerbach, } \\
\text { Bauer, } \\
\text { Reichert } \\
\text { (2008) }\end{array}$ & & & & Modelamiento & $\begin{array}{l}\text { Frecuencia } \\
\text { y selección }\end{array}$ & $\begin{array}{l}\text { Ejecución y } \\
\text { monitoreo }\end{array}$ & Optimización \\
\hline $\begin{array}{l}\text { Netjes, Reijers, } \\
\text { y Van der Aalst } \\
(2006)\end{array}$ & & & Diseño & Configuración & Ejecución & Control & Diagnóstico \\
\hline $\begin{array}{l}\text { Houy, Fettke } \\
\text { y Loos (2010) }\end{array}$ & & $\begin{array}{l}\text { Desarrollo de } \\
\text { estrategia }\end{array}$ & $\begin{array}{l}\text { Definición } \\
\text { y modelamiento }\end{array}$ & Implementación & Ejecución & $\begin{array}{l}\text { Monitoreo } \\
\text { y control }\end{array}$ & $\begin{array}{l}\text { Optimización y } \\
\text { mejoramiento }\end{array}$ \\
\hline $\begin{array}{l}\text { Muehlen } \\
\text { y Ho (2006) }\end{array}$ & & $\begin{array}{l}\text { Especificación } \\
\text { de objetivos } \\
\text { y análisis del } \\
\text { entorno }\end{array}$ & Diseño & Implementación & Monitoreo & Evaluación & \\
\hline $\begin{array}{l}\text { Van der Aalst } \\
(2004)\end{array}$ & & & Diseño & Configuración & Ejecución & Diagnóstico & \\
\hline Verma (2009) & & $\begin{array}{l}\text { Definición de } \\
\text { objetivos }\end{array}$ & $\begin{array}{l}\text { Identificación de } \\
\text { procesos }\end{array}$ & $\begin{array}{l}\text { Clasificación de } \\
\text { procesos }\end{array}$ & $\begin{array}{l}\text { Selección de } \\
\text { procesos }\end{array}$ & $\begin{array}{l}\text { Definición de } \\
\text { herramientas e } \\
\text { implementación }\end{array}$ & $\begin{array}{l}\text { Monitoreo } \\
\text { de procesos }\end{array}$ \\
\hline Weske (2007) & & $\begin{array}{l}\text { Administración } \\
\text { de stakeholders }\end{array}$ & Diseño y análisis & Configuración & Operación & $\begin{array}{l}\text { Evaluación de } \\
\text { desempeño }\end{array}$ & \\
\hline $\begin{array}{l}\text { El Kharbili } \\
\text { y Keil (2010) }\end{array}$ & & & $\begin{array}{l}\text { Análisis de } \\
\text { reglas de } \\
\text { procesos de } \\
\text { negocio }\end{array}$ & $\begin{array}{l}\text { Modelamiento } \\
\text { de reglas de } \\
\text { procesos de } \\
\text { negocio }\end{array}$ & $\begin{array}{l}\text { Implementación } \\
\text { de reglas de } \\
\text { negocio }\end{array}$ & & \\
\hline Šaša (2010) & $\begin{array}{l}\text { Captura de } \\
\text { conocimiento } \\
\text { de toma de } \\
\text { decisión }\end{array}$ & & & & $\begin{array}{l}\text { Automatización } \\
\text { de las actividades } \\
\text { de toma de } \\
\text { decisión }\end{array}$ & & \\
\hline $\begin{array}{l}\text { Gong y } \\
\text { Janssen (2012) }\end{array}$ & $\begin{array}{l}\text { Adaptación } \\
\text { de política de } \\
\text { negocio }\end{array}$ & & & & $\begin{array}{l}\text { Implementación } \\
\text { de las políticas de } \\
\text { negocio }\end{array}$ & & \\
\hline $\begin{array}{l}\text { Tran et al. } \\
\text { (2012) }\end{array}$ & $\begin{array}{l}\text { Modelo de } \\
\text { cumplimiento }\end{array}$ & & & & $\begin{array}{l}\text { Automatización } \\
\text { de asuntos } \\
\text { relacionados } \\
\text { al cumplimiento } \\
\text { de las leyes }\end{array}$ & & \\
\hline
\end{tabular}


(continuación)

\begin{tabular}{|c|c|c|c|c|c|c|c|}
\hline \multirow{2}{*}{$\begin{array}{l}\text { Referencia } \\
\text { Isaca (2012) }\end{array}$} & \multicolumn{7}{|c|}{ Actividades } \\
\hline & $\begin{array}{l}\text { Definición de } \\
\text { requerimientos }\end{array}$ & & $\begin{array}{l}\text { Identificar } \\
\text { soluciones }\end{array}$ & & $\begin{array}{l}\text { Construir las } \\
\text { soluciones }\end{array}$ & & \\
\hline $\begin{array}{l}\text { Scheer y } \\
\text { Brabänder } \\
(2010)\end{array}$ & & Estrategia & & Diseño & Implementación & Control & \\
\hline $\begin{array}{l}\text { Duarte et al. } \\
\text { (2012) }\end{array}$ & $\begin{array}{l}\text { Selección de } \\
\text { modelo de } \\
\text { proceso }\end{array}$ & & & $\begin{array}{l}\text { Especificación } \\
\text { de modelo de } \\
\text { proceso }\end{array}$ & $\begin{array}{l}\text { Implementación } \\
\text { del modelo de } \\
\text { proceso }\end{array}$ & & \\
\hline $\begin{array}{l}\text { Fabra et al. } \\
(2012)\end{array}$ & & & $\begin{array}{l}\text { Modelamiento } \\
\text { de proceso de } \\
\text { negocio }\end{array}$ & $\begin{array}{l}\text { Modelamiento } \\
\text { de casos de uso } \\
\text { y de servicios }\end{array}$ & $\begin{array}{l}\text { Composición de } \\
\text { servicios }\end{array}$ & & \\
\hline $\begin{array}{l}\text { Lederer et al. } \\
\text { (2013) }\end{array}$ & $\begin{array}{l}\text { Estrategia de } \\
\text { negocio }\end{array}$ & & $\begin{array}{l}\text { Análisis de } \\
\text { procesos de } \\
\text { negocio }\end{array}$ & & & & \\
\hline $\begin{array}{l}\text { Santos et al. } \\
\text { (2013) }\end{array}$ & & & & $\begin{array}{l}\text { Diseño de } \\
\text { proceso }\end{array}$ & $\begin{array}{l}\text { Implementación } \\
\text { del proceso }\end{array}$ & & \\
\hline $\begin{array}{l}\text { Gulledge } \\
\text { (2015) }\end{array}$ & & & Descubrir & Diseñar & Construir & & \\
\hline $\begin{array}{l}\text { Ndie et al. } \\
(2010)\end{array}$ & & & Análisis MDA & & $\begin{array}{l}\text { Implementación } \\
\text { MDA }\end{array}$ & & \\
\hline $\begin{array}{l}\text { Appel, Kleber, } \\
\text { Frischbier, } \\
\text { Freudenreich } \\
\text { y Buchmann } \\
\text { (2014) }\end{array}$ & $\begin{array}{l}\text { Transición } \\
\text { desde el } \\
\text { modelamiento } \\
\text { del proceso } \\
\text { hasta la } \\
\text { ejecución del } \\
\text { proceso }\end{array}$ & & & & & & \\
\hline $\begin{array}{l}\text { Zhu, X., } \\
\text { vanden } \\
\text { Broucke, Zhu, } \\
\text { G., Vanthienen } \\
\text { y Baesens } \\
(2016)\end{array}$ & $\begin{array}{l}\text { Gobierno de } \\
\text { las actividades } \\
\text { de modelado y } \\
\text { ejecución }\end{array}$ & & & & & & \\
\hline $\begin{array}{l}\text { Brocke, Zelt } \\
\text { y Schmiedel } \\
(2015)\end{array}$ & $\begin{array}{l}\text { Identificación } \\
\text { del contexto en } \\
\text { el que se aplica } \\
\text { para la gestión } \\
\text { de procesos de } \\
\text { negocio }\end{array}$ & & & & & & \\
\hline $\begin{array}{l}\text { Cabanillas, } \\
\text { Resinas, Del } \\
\text { Río y Ruiz } \\
\text { (2015) }\end{array}$ & $\begin{array}{l}\text { Automatización } \\
\text { del análisis del } \\
\text { proceso }\end{array}$ & & & & & & \\
\hline
\end{tabular}


La tabla 2 presenta el porcentaje de procesos de automatización de procesos de negocio que realizan las actividades de referencia propuesta por la ABPMP (2009).

Tabla 2. Tasa de ejecución de actividades

\begin{tabular}{|c|c|}
\hline Actividad & $\%$ \\
\hline Planeamiento y estrategia & 0,22 \\
\hline Análisis & 0,52 \\
\hline Diseño y modelamiento & 0,57 \\
\hline Implementación & 0,78 \\
\hline Monitoreo y control & 0,35 \\
\hline Refinamiento & 0,17 \\
\hline No Actividad & 0,48 \\
\hline
\end{tabular}

Fuente: ABPMP (2009)

Se observa que las actividades del proceso de automatización de procesos de negocio tienen muy poca similitud con relación al proceso de referencia propuesto por la ABPMP (2009). En la mayoría de los procesos de automatización de procesos de negocio se realiza la actividad de implementación.

No hay estandarización de las actividades en los procesos de automatización de procesos de negocio, la cual es de utilidad para mejorar la orientación de los esfuerzos para el desarrollo de recursos que faciliten la ejecución de cada una de las actividades del proceso de automatización de procesos de negocio; por ejemplo, metamodelos de procesos de negocio, notaciones para el modelamiento de procesos de negocio, herramientas de transformación de modelos de procesos de negocio, la transformación de BPMN a UML.

\subsection{Q2 ¿Cuál es el nivel de integración entre las actividades del proceso de automatización de procesos de negocio?}

Una empresa integrada coordina sus estrategias, sus tácticas y sus decisiones para una implementación oportuna y eficiente del flujo de información; y una estructura de la organización que permita usar esta información para un control físico óptimo. La integración horizontal consiste en la integración lógica y física del proceso de negocio desde la demanda del producto hasta la distribución, considerando el flujo de material y de documentos $y$, por lo tanto, dependiente de la tecnología. La integración vertical incorpora a los tomadores de decisión, a los sistemas de información para la toma de decisiones, y al flujo de decisión (Vernadat, 1996).

El proceso de automatización de procesos de negocio involucra a los estrategas de negocio (Scheer y Brabänder, 2010); a los especialistas de tecnologías de información, 
quienes son los stakeholders de la automatización de procesos de negocio; y a los analistas de negocio (Fabra et al., 2012). El proceso de automatización de procesos de negocio requiere un control de inicio a fin; es decir, una integración horizontal (Vernadat, 1996). Los niveles de integración entre las actividades del proceso de automatización de procesos de negocio toman los valores de 0 a 3 que corresponden a nivel de integración ninguna, parcial, completa e integrada, tal como se aprecia en la tabla 3.

\section{Tabla 3. Nivel de integración}

\begin{tabular}{|c|l|l|}
\hline \multicolumn{2}{|c|}{ Vivel } & \multicolumn{2}{c|}{ Descripción } \\
\hline 0 & Ninguna & La información recibida no es parte de una base de base de datos \\
\hline 1 & Parcial & La información recibida es parcialmente parte de una base de base de datos \\
\hline 2 & Completa & $\begin{array}{l}\text { La información recibida es parte de una base de base de datos y requiere ser } \\
\text { trasformada }\end{array}$ \\
\hline 3 & Integrada & $\begin{array}{l}\text { La información recibida es parte de una base de base de datos y no requiere ser } \\
\text { transformada }\end{array}$ \\
\hline
\end{tabular}

Elaboración propia

La integración entre las actividades de los procesos de automatización de procesos de negocio se mide por la forma de compartir la información entre una actividad y otra. Los procesos de automatización de procesos de negocio sometidos al análisis de integración se muestran en la tabla 4.

Tabla 4. Procesos de automatización de procesos de negocio

\begin{tabular}{|c|c|c|}
\hline Id & Autor(es) & Proceso de automatización de procesos de negocio \\
\hline P1 & El Kharbili y Keil (2010) & Modelo de reglas de decisiones integrados a los procesos de negocio \\
\hline P2 & Šaša (2010) & $\begin{array}{l}\text { Modelo para la automatización de las actividades de toma de decisión basados } \\
\text { en ontologías y tecnologías de agentes }\end{array}$ \\
\hline P3 & Tran et al. (2012) & $\begin{array}{l}\text { Modelo integrado para la automatización de asuntos relacionados } \\
\text { al cumplimiento de las leyes }\end{array}$ \\
\hline P4 & Fabra et al. (2012) & Modelo integrado de desarrollo orientado a servicios \\
\hline P5 & Santos et al., (2013) & $\begin{array}{l}\text { Modelo enfocado en la transformación para obtener un modelo ejecutable } \\
\text { para el proceso de negocio }\end{array}$ \\
\hline P6 & Duarte et al. (2012) & Modelo de implementación de negocio \\
\hline P7 & Lederer et al. (2013) & $\begin{array}{l}\text { Modelo de alineación de la estrategia de negocio con métodos de análisis } \\
\text { de proceso de negocio }\end{array}$ \\
\hline P8 & Isaca (2012) & $\begin{array}{l}\text { Modelo de alineamiento de los objetivos de tecnologías de la información } \\
\text { con los objetivos del negocio }\end{array}$ \\
\hline P9 & Ndie et al. (2010) & Modelo de la arquitectura conducido por modelos \\
\hline $\mathrm{P} 10$ & Gong y Janssen (2012) & Modelo de implementación de las políticas de negocio \\
\hline
\end{tabular}


Para el análisis de la integración de proceso de automatización de procesos de negocio se han tomado como referencia las actividades de los procesos de gestión de proceso de negocio propuesto por la ABPMP (2009) (tabla 5).

Tabla 5. Niveles de integración entre las actividades de los procesos de automatización de procesos de negocio

\begin{tabular}{|l|c|c|c|c|c|c|c|c|c|c|}
\hline \multicolumn{10}{|c|}{ Procesos de automatización de procesos de negocio } \\
\hline \multicolumn{1}{|c|}{ Actividad } & P1 & P2 & P3 & P4 & P5 & P6 & P7 & P8 & P9 & P10 \\
\hline $\begin{array}{l}\text { Planeamiento } \\
\text { y estrategia }\end{array}$ & 2 & 0 & 1 & 0 & 0 & 1 & 2 & 2 & 0 & 1 \\
\hline Análisis & 0 & 0 & 0 & 0 & 0 & 0 & 0 & 0 & 0 & 0 \\
\hline $\begin{array}{l}\text { Diseño } \\
\text { y modelamiento }\end{array}$ & 0 & 0 & 1 & 1 & 1 & 1 & 0 & 0 & 0 & 0 \\
\hline $\begin{array}{l}\text { Implementación } \\
\text { Monitoreo }\end{array}$ & 0 & 2 & 2 & 2 & 2 & 2 & 0 & 0 & 2 & 0 \\
\hline y control & 0 & 0 & 0 & 0 & 0 & 0 & 0 & 0 & 0 & 0 \\
\hline Refinamiento & 0 & 0 & 0 & 0 & 0 & 0 & 0 & 0 & 0 & 0 \\
\hline
\end{tabular}

Se puede observar que ninguno de los procesos de automatización de procesos de negocio (P1 a P10) tiene sus actividades integradas. Los valores de integración de algunas de sus actividades están entre 0 y 2 . Esto quiere decir que no hay integración entre las actividades de los procesos de automatización de inicio a fin. Es muy importante la integración entre las actividades de los procesos de automatización de inicio a fin, pues ello permitiría un proceso de automatización de procesos de negocio más rápido y más eficiente (Vernadat, 1996).

\subsection{Q3 ¿Qué métodos del proceso de automatización de procesos de negocio se usan en cada una de las actividades del proceso de automatización de procesos de negocio?}

La arquitectura dirigida por modelos (MDA) es un acercamiento al diseño de software, propuesto y patrocinado por el Object Management Group. MDA se ha concebido para dar soporte a la ingeniería dirigida a modelos de los sistemas de software. MDA propone un marco de trabajo para una arquitectura, y brinda un conjunto de guías para estructurar especificaciones expresadas como modelos. 
Tabla 6. Métodos de los procesos de automatización de procesos de negocio

\begin{tabular}{|c|c|c|c|}
\hline Autor & Tipo de método & Descripción & Herramienta \\
\hline Bezivin (2003) & MDD & $\begin{array}{l}\text { Diagramas de actividad UML } \\
2 \text { - PIM a BPEL- PSM }\end{array}$ & $\begin{array}{l}\text { Lenguaje de transformación Atlas } \\
\text { (ATL) }\end{array}$ \\
\hline White (2005) & MDD & BPMN 1 a WS-BPEL & \\
\hline \multirow[t]{2}{*}{ Lezoche (2005) } & MDD & CPE - CIM a BPMN - PIM & \\
\hline & MDD & BPMN - PIM a BPEL- PSM & \\
\hline Koehler (2005) & MDD & UML 2 - PIM a BPEL- PSM & $\begin{array}{l}\text { Lenguaje de restricciones de } \\
\text { objetos }(O C L)\end{array}$ \\
\hline Brambilla (2006) & MDD & $\begin{array}{l}\text { BPMN a Diagramas } \\
\text { hipertexto WebML }\end{array}$ & \\
\hline Zhao (2006) & MDD & UML 2 - PIM a BPEL- PSM & $\begin{array}{l}\text { Lenguaje de expresiones } \\
\text { regulares (REL) }\end{array}$ \\
\hline $\begin{array}{l}\text { Rungworawut } \\
(2006)\end{array}$ & MDD & BPMN - CIM a UML - PIM & $\begin{array}{l}\text { Diagramas de casos de uso o } \\
\text { diagramas de clase }\end{array}$ \\
\hline Dijkman (2010) & MDD & BPMN 2.0 & Sistema de workflow YAWL \\
\hline $\begin{array}{l}\text { Meertens et al. } \\
(2012)\end{array}$ & MDD & $\begin{array}{l}\text { Modelo de negocio Canvas } \\
\text { a Arquitectura empresarial } \\
\text { ArchiMate }\end{array}$ & \\
\hline Appel et al. (2014) & $\begin{array}{l}\text { Unidad de } \\
\text { procesamiento de } \\
\text { flujo de eventos - } \\
\text { UPFE }\end{array}$ & $\begin{array}{l}\text { Transformación de UPFE en } \\
\text { EPC a UPFE en BPMN }\end{array}$ & ARIS \\
\hline $\begin{array}{l}\text { Cabanillas et al. } \\
\text { (2015) }\end{array}$ & $\begin{array}{l}\text { Automatización del } \\
\text { análisis del proceso }\end{array}$ & Modelo de negocio & Lógica descriptiva \\
\hline
\end{tabular}

MDD: Model Driven Development (desarrollo dirigido por modelos)

Elaboración propia

Hay una tendencia al uso de MDA para la automatización de procesos de negocio utilizando herramientas de transformación de modelos, como se puede ver en la tabla 6 . La transformación automática de los modelos de procesos de negocio es muy importante, porque permite mejorar la comunicación entre los estrategas de negocio, analistas de negocio y especialistas de tecnologías de información (stakeholders de automatización de procesos de negocio), lo que trae como consecuencia la reducción de riesgos de errores en la especificación de los modelos de procesos, y también evita la duplicidad de esfuerzos de los stakeholders de procesos de negocio para realizar los modelos de procesos de negocio (Fabra et al., 2012) (Heininger, 2012) (Duarte et al., 2012) (Santos et al., 2013). La tendencia de la aplicación de la arquitectura conducidos por modelos es para las actividades de diseño e implementación con modelos BPMN y BPEL, respectivamente.

Resulta conveniente la aplicación de la arquitectura conducida por modelos para todas las actividades del proceso de automatización de procesos de negocio, pues esto permitiría la 
trazabilidad en todo el proceso de automatización de procesos de negocio y, por consiguiente, mejoraría la comunicación entre los stakeholders de automatización de procesos de negocio.

Por lo tanto, la aplicación de MDA en todo el proceso ciclo de vida de automatización de procesos de negocio posibilitaría que el proceso de automatización de procesos de negocio sea más rápido y más eficiente.

\subsection{Q4 ¿Qué modelos de procesos de negocio se usan en cada una de las actividades del proceso de automatización de procesos de negocio?}

Los modelos de procesos de negocio tienen un propósito y se describen con la herramienta de modelado correspondiente (CPE, BPMN, BPEL, UML, etcétera.) (Becker, Kugeler y Rosemann, 2003). Los procesos de automatización de procesos de negocio (ver tabla 6) realizan las actividades de diseño e implementación con modelos BPMN y BPEL, respectivamente.

En el proceso de automatización de procesos de negocio es importante la realización de la actividad de estrategia (Scheer y Brabänder, 2010) o la actividad de planeamiento y estrategia (ABPMP, 2009) para garantizar el éxito del proceso de negocio automatizado (Meertens et al., 2012). Asimismo, para lograr la integración de inicio a fin del proceso de automatización de procesos de negocio (Vernadat, 1996).

\subsection{Q5 ¿Qué objetivos e indicadores se usan en el proceso de automatización de procesos de negocio y en cada una de las actividades del proceso de automatización de los procesos de negocio?}

En los procesos de automatización de procesos de negocio revisados (ver tabla 4) no se establecen objetivos e indicadores en los procesos de automatización de procesos de negocio y sus actividades. Es fundamental la determinación de los objetivos e indicadores del proceso de automatización de procesos de negocio y de las actividades que se realizan en este proceso, ya que sin ellos no sería posible gestionar el proceso de automatización de procesos de negocio.

\section{Conclusiones}

Se ha logrado el propósito de organizar la información recopilada acerca de los procesos de automatización de procesos de negocio para analizar las tendencias y variabilidad de los elementos del proceso de automatización de procesos de negocio. Luego de la revisión y organización realizada de la literatura existente, se presentan las siguientes conclusiones:

i. Los procesos de automatización de procesos de negocio (ver tabla 1) tienen diferentes actividades. Es decir, no están estandarizados. 
ii. No hay integración entre las actividades del proceso de automatización de procesos de negocio (ver tabla 5).

iii. Existe una tendencia del uso del método de automatización de desarrollo conducido por modelos - MDD (ver tabla 6).

iv. Las herramientas de modelado utilizadas por las actividades de los procesos de automatización de procesos de negocio son BPMN y BPEL (ver tabla 6).

v. No se establecieron objetivos ni indicadores para los procesos de automatización de procesos de negocio, ni para sus actividades (ver tabla 4).

\section{Referencias}

Appel, S., Kleber, P., Frischbier, S., Freudenreich, T., y Buchmann, A. (2014). Modeling and execution of event stream processing in business processes. Information Systems, 46, 140-156. DOI: $10.1016 /$ j.is.2014.04.002

Association of Business Process Management Professionals, ABPMP (2009). Guide to the Business Process Management Common Body of Knowledge. Florida, Estados Unidos: Autor

Becker, J., Kugeler, M., y Rosemann, M. (eds.) (2003). Process management: A guide for the design of business processes. Berlín: Springer.

Brocke, J., Zelt, S., y Schmiedel, T. (2015). On the role of context in business process management. International Journal of Information Management, 36(3), 486-495. DOI: 10.1016/j. ijinfomgt.2015.10.002

Burlton, R. (2010). Delivering business strategy through process management. Handbook on Business Process Management 2 (45-78). Berlín, Alemania: Springer-Verlag. DOI: 10.1007/978-3-642-45103-4_2

Burlton, T. (2001). Business process management profiting from process. Indianapolis, Estados Unidos: SAMS.

Cabanillas, C., Resinas, M., Del Río, A., y Ruiz, A. (2015). Specification and automated design-time analysis of the business process human resource perspective. Information Systems, 52, 55-82. DOI: 10.1016/j.is.2015.03.002

Davenport, T. (1993). Process innovation, reengineering work through information technology. Boston, Estados Unidos: Harvard Business School Press. 
Duarte, F., Machado, R., y Fernandes, J. (2012). BIM: A methodology to transform business processes into software systems (39-58). Berlín, Alemania: Springer-Verlag. DOI: 10.1007/978-3-642-27213-4_4

El Kharbili, M., y Keil, T. (2010). Bringing agility to Business Process Management: Rules deployment in an SOA. Emerging Web Services Technology Volume III (157-170). Basilea, Suiza: Birkhäuser. DOI: 10.1007/978-3-0346-0104-7_11

Fabra, J., De Castro, V., Álvarez, P., y Marcos, E. (2012). Automatic execution of business process models: Exploiting the benefits of Model-driven Engineering approaches. Journal of Systems and Software, 85(3), 607-625. DOI: 10.1016/j.jss.2011.09.022

Gong, Y., y Janssen, M. (2012). From policy implementation to business process management: Principles for creating flexibility and agility. Government Information Quarterly, 29, S61-S71. DOI: 10.1016/j.giq.2011.08.004

Gulledge, T. (2015). Integrated Business Process and Service Management. Handbook on Business Process Management 1 (579-594). Berlín, Alemania: Springer-Verlag. DOI: 10.1007/978-3-642-45100-3_25

Hallerbach, A., Bauer, T., y Reichert, M. (2008). Managing process variants in the process life cycle. 10th International Conference on Enterprise Information Systems, 154-161.

Heininger, R. (2012). Requirements for Business Process Management Systems Supporting Business Process Agility. En S. Oppl, y A. Fleischmann (eds.). S-BPM ONE - Education and Industrial Developments. (168-180). DOI: 10.1007/978-3-642-29294-1_12

Houy, C., Fettke, P., y Loos, P. (2010). Empirical research in business process management - analysis of an emerging field of research. Business Process Management Journal, 16(4), 619-661. DOI: $10.1108 / 14637151011065946$

Isaca (2012). COBIT 5: A Business Framework for the Governance and Management of Enterprise IT. Illinois, Estados Unidos: Autor.

Lederer, M., Schott, P., Huber, S., y Kurz, M. (2013). Strategic Business Process Analysis: A Procedure Model to Align Business Strategy with Business Process Analysis Methods (pp. 247-263). DOI: 10.1007/978-3-642-36754-0_16

Macedo, R., Kazan, S., Dallavalle, S., y Lucirton, A. (2014). An analysis of BPM lifecycles: from a literature review to a framework proposal. Business Process Management Journal, 20(3), 412-432. DOI: 10.1108/BPMJ-03-2013-0035 
Meertens, L., lacob, M., Nieuwenhuis, L., van Sinderen, M., Jonkers, H., y Quartel, D. (2012). Mapping the business model canvas to ArchiMate. Proceedings of the 27th Annual ACM Symposium on Applied Computing - SAC'12 (1694-1701). Nueva York, Estados Unidos: ACM Press. DOI: $10.1145 / 2245276.2232049$

Muehlen, M., y Ho, D. (2006). Risk Management in the BPM Lifecycle. Lecture Notes in Computer Science-BPM 2005 Workshops, 3812, 454-466. DOI: 10.1007/11678564_42

Ndie, T., Tangha, C., y Ekwoge, F. (2010). MDA (Model-Driven Architecture) as a software industrialization pattern: An approach for a pragmatic software factories. Journal of Software Engineering and Applications, 3(6), 561-571. DOI: 10.4236/jsea.2010.36065

Netjes, M., Reijers, H., y van der Aalst, W. (2006). Supporting the BPM life-cycle with FileNet. Workshop on Exploring Modeling Methods for Systems Analysis and Design (EMMSAD'06), held in conjunctiun with the 18th Conference on Advanced Information Systems (CAiSE'06), 497-508. Namur, Bélgica: Namur University Press.

Santos, N., Duarte, F., Machado, R., y Fernandes, J. (2013). A transformation of Business Process Models into Software-Executable Models using MDA (147-167). Berlín, Alemania: SpringerVerlag. DOI: 10.1007/978-3-642-35702-2_10

Šaša, A. (2010). A Model for Business Process Automation in Service Oriented Systems with Knowledge Management Technologies. 20106 th World Congress on Services (136-139). IEEE. DOI: 10.1109/SERVICES.2010.71

Scheer, A., y Brabänder, E. (2010). The process of Business Process Management. Handbook on Business Process Management 2 (239-265). Berlín, Alemania: Springer-Verlag. DOI: 10.1007/978-3-642-01982-1_12

Tran, H., Zdun, U., Holmes, T., Oberortner, E., Mulo, E., y Dustdar, S. (2012). Compliance in service-oriented architectures: A model-driven and view-based approach. Information and Software Technology, 54(6), 531-552. DOI: 10.1016/j.infsof.2012.01.001

Verma, N. (2009). Business Process Management: Profiting from process. Nueva Delhi, India: Global India.

Vernadat, F. (1996). Enterprise modeling and integration: Principles and applications. Londres, Inglaterra: Chapman \& Hall.

Weske, M. (2007). Business Process Management. (2007). Berlín, Alemania: Springer-Verlag. DOI: 10.1007/978-3-540-73522-9

Zhu, X., van den Broucke, S., Zhu, G., Vanthienen, J., y Baesens, B. (2016). Enabling flexible location-aware business process modeling and execution. Decision Support Systems, 83, 1-9. DOI: $10.1016 /$ j.dss.2015.12.003 